\title{
THE OSCILLATION OF SOLUTIONS OF VOLTERRA INTEGRAL AND INTEGRO-DIFFERENTIAL EQUATIONS WITH HIGHLY OSCILLATORY KERNELS
}

\author{
HERMANN BRUNNER, YUNYUN MA AND YUESHENG XU
}

Communicated by Terry Herdman

\begin{abstract}
We study the oscillatory structures of solutions of Volterra integral and integro-differential equations (VIEs, VIDEs) with highly oscillatory kernels. Based on the structured oscillatory spaces introduced in Wang and $\mathrm{Xu}$ [28], we first analyze the degree of oscillation of the solution of VIEs associated with the oscillatory kernels belonging to a certain structured oscillatory space by using the resolvent representation of the solution. According to a decomposition of the oscillatory integrals in the complex plane, we prove that the Volterra integral operator reduces the oscillatory order of the functions in the structured oscillatory spaces corresponding to the oscillatory structure of the kernel. The analogous oscillatory structure of solutions of VIDEs is then analyzed by representing the solution of the VIDEs by the differential resolvent kernel and by exploiting the relationship between the VIDEs and the equivalent VIE. We conclude that the solutions of the VIEs and VIDEs preserve the oscillatory components of the kernel.
\end{abstract}

1. Introduction. We consider, in this paper, the qualitative theory of linear Volterra integral and integro-differential equations (VIEs and VIDEs) with highly oscillatory kernels whose oscillators are nonlinear separable functions. The presence of oscillations in the kernels leads to

2010 AMS Mathematics subject classification. Primary 45D05, 45H05, 45J05.

Keywords and phrases. Volterra integral equation, Volterra integro-differential equation, highly oscillatory kernel, oscillatory structured space, decomposition of the oscillatory integral, oscillation preserving solution.

This research is supported in part by the Hong Kong Research Grants Council (HKBU No. 200113), the Natural Sciences Engineering Research Council of Canada (Discovery grant No. A9406), the U.S. National Science Foundation under grant No. DMS-152233, the Guangdong Provincial Government of China through the Computational Science Innovative Research Team program and the Natural Science Foundation of China under grant No. 11471013. The third author is the corresponding author.

Received by the editors on June 12, 2015. 
an oscillatory behavior of the solutions of these equations. A thorough understanding of this oscillatory behavior of the solution is crucial for the analysis and the design of the numerical methods that preserve these oscillatory properties.

VIEs and VIDEs arise in applications as mathematical models of various physical processes and biological phenomena $[\mathbf{1}, \mathbf{3}, \mathbf{1 6}]$. The most comprehensive and advanced analyses of the VIEs and VIDEs are contained in the monographs $[\mathbf{2}, \mathbf{1 0}, \mathbf{2 0}]$. The theory of the VIEs with highly oscillatory kernel was presented in [3], where the technique based on integration by parts was used to derive the asymptotic expansion of the solution in terms of inverse powers of the wavenumber. The development of numerical approximations to solutions of highly oscillatory VIEs can be found in $[\mathbf{2 7}, \mathbf{3 0}, \mathbf{3 1}]$. These numerical methods were constructed with the help of the techniques for computing the oscillatory integrals. However, these methods do not consider the influence of the highly oscillatory behavior of the solutions. Therefore, the numerical approximation of the solution to the VIEs and VIDEs in the appropriate oscillatory spaces remains a challenging problem.

We now review the recent developments of Fredholm integral equations (FIE) with oscillatory kernels. They are relevant for the study of VIEs, since a Fredholm integral operator can be split into two Volterra integral operators, and Volterra integral operators may be viewed as Fredholm integral operators with special kernels. In [26], the asymptotic behavior of the solutions, as the wavenumber tends to infinity, was analyzed, and it was shown that the maximum norm of the solution just depends on the right-hand side of the equations. In [5], the spectral problem of FIEs with oscillatory kernels is studied, and the computation of the spectral is considered in [4]. The recent paper [28] leads to a new method for analyzing the oscillatory integral equations based on oscillatory function spaces and iterated integral operators. It first introduces the notion of the degree of oscillation of an oscillatory function and then explores the oscillatory properties of the solutions of the equations in the oscillatory structured spaces of Wang-Xu constructed by the oscillatory components of the kernel. The oscillation-preserving Galerkin method was proposed in [28] for the numerical approximation of the solution by incorporating the standard approximation subspaces with a finite number of oscillatory functions. 
We remark that the methods for calculating oscillatory integrals play an important role in analyzing the theory of oscillatory integral operators such as Fredholm integral operators and Volterra integral operators, and the numerical calculations for oscillatory integral equations. Highly oscillatory integrals are now well understood, and many highly efficient methods have been devised so that the accuracy of the computation increases as the wavenumber tends to infinity. There are mainly four classes of methods: asymptotic [13, 25], Filon-type $[\mathbf{8}, \mathbf{9}, \mathbf{1 3}, \mathbf{1 4}, \mathbf{2 9}$, Levin-type $[\mathbf{1 7}, \mathbf{1 8}, \mathbf{2 1}, \mathbf{2 2}]$ and numerical steepest descent $[\mathbf{1 1}, \mathbf{1 2}]$. A new method proposed in [19] is more efficient than most of the existing methods, as it combines the moment free Filon-type methods with meshes that are graded according to the wavenumber. There are three techniques used to analyze and compute the oscillatory integrals: integration by parts, changing the interval of integration into the paths in the complex plane, and graded meshes depending on the wavenumber. In $[\mathbf{3}, \mathbf{2 8}]$, the first technique is used to derive the oscillatory components of integral operators.

The purpose of this paper is to explore the oscillatory structure of the solutions of VIEs and VIDEs with highly oscillatory kernels. The motivation for writing this paper is that, in contrast to Fredholm integral equations, the analysis of the oscillatory structure of solutions to Volterra integral and integro-differential equations is based on different techniques and has so far not been analyzed in detail. The results of our analysis will also play an important role in the design of efficient and highly accurate numerical schemes for such problems. Exploiting the relationship between VIDEs and VIEs, we analyze the oscillatory properties of the solutions of VIEs with kernels composed of two parts: a non-oscillatory smooth function and a product of a non-oscillatory smooth function and a typical known oscillatory function with nonlinear oscillator. The right-hand side and the coefficient function of the equations may have the same oscillations as the structure of the kernel. Based on the notions of oscillatory functions and appropriate oscillatory spaces defined in [28], we establish the degree of oscillation of the solutions to the VIEs and VIDEs by using the iterated kernels of the equations (cf., [2]). We derive the oscillatory components of the solutions by representing the solutions in terms of iterated integral operators as [28]. The decomposition of the oscillatory function is constructed by changing the interval of integration into a path in the 
complex plane, which is different from [28]. The solution is proved to be in the structured oscillatory spaces whose specific oscillatory structure is similar to that of the kernel.

The outline of this paper is as follows. We consider the VIEs in Sections 2 and 3. In Section 2, we derive the oscillatory degree of the resolvent kernel and the solution of the VIEs by utilizing the properties of Picard iteration kernels. The oscillatory structure of the solution to the VIEs is analyzed in Section 3. In Section 4, the analysis of Section 3 is extended to VIDEs. The final section of the paper describes our future work.

2. The degree of oscillation of solutions to VIEs. We investigate in this section the degree of oscillation of the solution of VIEs. This is done by a careful study of the resolvent kernel associated with the given kernel of the Volterra integral operator.

We begin by studying the relationship between the bound of derivatives of the solutions to the VIEs and the powers of the wavenumber. Let

$$
I:=[0,1] \text { and } D:=\{(t, s) ; 0 \leq s \leq t \leq 1\} .
$$

For fixed $\kappa>1$, we consider the second-kind VIE

$$
u(t)=f(t)+\left(\mathcal{V}_{\kappa} u\right)(t), \quad t \in I,
$$

with Volterra integral operator $\mathcal{V}_{\kappa}: C(I) \rightarrow C(I)$ defined by

$$
\left(\mathcal{V}_{\kappa} \phi\right)(t):=\int_{0}^{t} K_{\kappa}(t, s) \phi(s) \mathrm{d} s,
$$

where $f \in C(I)$ and the kernel $K_{\kappa} \in C(D)$ is highly oscillatory (its precise structure will be made precise in Assumption 3.6). Using the notation introduced in [28], the analysis of the oscillatory properties of the solution of (2.1) will be based on the resolvent kernel $R_{\kappa}$ associated with the given kernel $K_{\kappa}$ and the corresponding representation of the solution.

We first give a brief review of the relevant theory of VIEs. For $n \in \mathbb{N}:=\{1,2, \ldots\}$, the iterated kernels of $K_{\kappa}(t, s)$ are given by

$$
H_{n+1}(t, s ; \kappa):=\int_{s}^{t} H_{1}(t, v ; \kappa) H_{n}(v, s ; \kappa) \mathrm{d} v, \quad \text { for }(t, s) \in D,
$$


where $H_{1}(t, s ; \kappa):=K_{\kappa}(t, s)$, for $(t, s) \in D$. The resolvent kernel $R_{\kappa}$ associated with the given kernel $K_{\kappa}$ is defined by the Neumann series

$$
R_{\kappa}(t, s):=\sum_{n \in \mathbb{N}} H_{n}(t, s ; \kappa), \quad \text { for }(t, s) \in D,
$$

which converges absolutely and uniformly on $D$. Hence, if $K_{\kappa} \in C(D)$, then for any $f \in C(I)$ the VIE (2.1) has a unique solution $u \in C(I)$ of the form

$$
u(t)=f(t)+\int_{0}^{t} R_{\kappa}(t, s) f(s) \mathrm{d} s, \quad t \in I .
$$

We next focus on analyzing the properties of the resolvent kernel $R_{\kappa}$ defined by (2.4). To this end, we first show the structure of the iterated kernels for the simple case where

$$
K_{\kappa}(t, s):=a+b \mathrm{e}^{\mathrm{i} \kappa(t-s)}, \quad(t, s) \in D,
$$

with constants $a, b \neq 0$. For $n \in \mathbb{N}_{0}:=\{0,1, \ldots\}$, let

$$
O_{n}(t, s ; \kappa):=\int_{s}^{t}(v-s)^{n} \mathrm{e}^{\mathrm{i} \kappa(s-v)} \mathrm{d} v, \quad(t, s) \in D .
$$

Set $\mathbb{Z}_{n}^{+}:=\{1,2, \ldots, n\}$ and $\mathbb{Z}_{n}:=\{0\} \cup \mathbb{Z}_{n}^{+}$. We present an auxiliary result in the following lemma.

Lemma 2.1. If $\kappa \neq 0$, then, for $n \in \mathbb{N}_{0}$ and $(t, s) \in D$,

$$
O_{n}(t, s ; \kappa)=\frac{n !}{(\mathrm{i} \kappa)^{n+1}}-\sum_{j \in \mathbb{Z}_{n}} \frac{n !}{(n-j) !(\mathrm{i} \kappa)^{j+1}}(t-s)^{n-j} \mathrm{e}^{\mathrm{i} \kappa(s-t)} \text {. }
$$

Proof. We prove this result by induction. Clearly, we have that

$$
O_{0}(t, s ; \kappa)=\left(1-\mathrm{e}^{\mathrm{i} \kappa(s-t)}\right) /(\mathrm{i} \kappa), \quad(t, s) \in D .
$$

Hence, the result of this lemma holds for $n=0$. We assume that this result is true for $n \in \mathbb{N}_{0}$ and consider the case $n+1$. Integration by parts leads to

$$
\begin{aligned}
O_{n+1}(t, s ; \kappa) & =-\frac{1}{\mathrm{i} \kappa}(t-s)^{n+1} \mathrm{e}^{\mathrm{i} \kappa(s-t)}+\frac{n+1}{\mathrm{i} \kappa} O_{n}(t, s ; \kappa) \\
& =\frac{(n+1) !}{(\mathrm{i} \kappa)^{n+2}}-\sum_{j \in \mathbb{Z}_{n+1}} \frac{(n+1) !(t-s)^{n+1-j} \mathrm{e}^{\mathrm{i} \kappa(s-t)}}{(n+1-j) !(\mathrm{i} \kappa)^{j+1}},
\end{aligned}
$$


for $(t, s) \in D$. We then obtain the conclusion of $n+1$. This completes the proof.

The next lemma shows the form of $H_{n}$ for $n \in \mathbb{N}$.

Lemma 2.2. For $n \in \mathbb{N}$, there exist $2 n$ constants $a_{n, j}$ and $b_{n, j}$ for $j \in \mathbb{Z}_{n}^{+}$independent of $\kappa$ such that, for $(t, s) \in D$,

$$
H_{n}(t, s ; \kappa)=\sum_{j \in \mathbb{Z}_{n}^{+}} a_{n, j} \frac{(t-s)^{j-1}}{(\mathrm{i} \kappa)^{n-j}}+\mathrm{e}^{\mathrm{i} \kappa(t-s)} \sum_{j \in \mathbb{Z}_{n}^{+}} b_{n, j} \frac{(t-s)^{j-1}}{(\mathrm{i} \kappa)^{n-j}} .
$$

Proof. We again use induction to establish this result. For $n=1$, it follows directly from the definition of $H_{1}$ with $a_{1,1}:=a$ and $b_{1,1}:=b$. Assume the result is true for $n \in \mathbb{N}$, and consider the case $n+1$. According to definition (2.3), we have, for $(t, s) \in D$, that

$$
\begin{aligned}
H_{n+1}(t, s ; \kappa) & \int_{s}^{t}\left(a_{1,1}+b_{1,1} \mathrm{e}^{\mathrm{i} \kappa(t-v)}\right) \\
\times & \left(\sum_{j \in \mathbb{Z}_{n}^{+}} a_{n, j} \frac{(v-s)^{j-1}}{(\mathrm{i} \kappa)^{n-j}}+\mathrm{e}^{\mathrm{i} \kappa(v-s)} \sum_{j \in \mathbb{Z}_{n}^{+}} b_{n, j} \frac{(v-s)^{j-1}}{(\mathrm{i} \kappa)^{n-j}}\right) \mathrm{d} v \\
= & \sum_{j \in \mathbb{Z}_{n}^{+}} a_{1,1} a_{n, j} \int_{s}^{t} \frac{(v-s)^{j-1}}{(\mathrm{i} \kappa)^{n-j}} \mathrm{~d} v+\mathrm{e}^{\mathrm{i} \kappa(t-s)} \sum_{j \in \mathbb{Z}_{n}^{+}} b_{1,1} b_{n, j} \int_{s}^{t} \frac{(v-s)^{j-1}}{(\mathrm{i} \kappa)^{n-j}} \mathrm{~d} v \\
& +\sum_{j \in \mathbb{Z}_{n}^{+}} a_{1,1} b_{n, j} \int_{s}^{t} \frac{(v-s)^{j-1}}{(\mathrm{i} \kappa)^{n-j}} \mathrm{e}^{-\mathrm{i} \kappa(s-v)} \mathrm{d} v \\
& +\mathrm{e}^{\mathrm{i} \kappa(t-s)} \sum_{j \in \mathbb{Z}_{n}^{+}} b_{1,1} a_{n, j} \int_{s}^{t} \frac{(v-s)^{j-1}}{(\mathrm{i} \kappa)^{n-j}} \mathrm{e}^{\mathrm{i} \kappa(s-v)} \mathrm{d} v .
\end{aligned}
$$

This together with the definitions of $O_{n}$ yields, for $(t, s) \in D$,

$$
\begin{aligned}
H_{n+1}(t, s ; \kappa)= & \sum_{j \in \mathbb{Z}_{n}^{+}} a_{1,1} a_{n, j} \frac{(t-s)^{j}}{j(\mathrm{i} \kappa)^{n-j}} \\
& +\mathrm{e}^{\mathrm{i} \kappa(t-s)} \sum_{j \in \mathbb{Z}_{n}^{+}} b_{1,1} b_{n, j} \frac{(t-s)^{j}}{j(\mathrm{i} \kappa)^{n-j}}
\end{aligned}
$$




$$
\begin{aligned}
& +\sum_{j \in \mathbb{Z}_{n}^{+}} a_{1,1} b_{n, j} \frac{O_{j-1}(t, s ;-\kappa)}{(\mathrm{i} \kappa)^{n-j}} \\
& +\mathrm{e}^{\mathrm{i} \kappa(t-s)} \sum_{j \in \mathbb{Z}_{n}^{+}} b_{1,1} a_{n, j} \frac{O_{j-1}(t, s ; \kappa)}{(\mathrm{i} \kappa)^{n-j}} .
\end{aligned}
$$

Applying Lemma 2.1, we find for $j \in \mathbb{Z}_{n}^{+}$and $(t, s) \in D$,

$$
\frac{O_{j-1}(t, s ; \kappa)}{(\mathrm{i} \kappa)^{n-j}}=\frac{(j-1) !}{(\mathrm{i} \kappa)^{n}}-\sum_{i \in \mathbb{Z}_{j-1}} \frac{(j-1) !(t-s)^{j-1-i} \mathrm{e}^{\mathrm{i} \kappa(s-t)}}{(j-1-i) !(\mathrm{i} \kappa)^{n-(j-1-i)}}
$$

and

$$
\frac{O_{j-1}(t, s ;-\kappa)}{(\mathrm{i} \kappa)^{n-j}}=\frac{(j-1) !}{(-\mathrm{i} \kappa)^{n}}-\sum_{i \in \mathbb{Z}_{j-1}} \frac{(j-1) !(t-s)^{j-1-i} \mathrm{e}^{\mathrm{i} \kappa(t-s)}}{(j-1-i) !(-\mathrm{i} \kappa)^{n-(j-1-i)}} .
$$

Substitution of the above two equations into (2.6) leads to the desired result.

Note that the resolvent kernel $R_{\kappa}$ of (2.1) has the same structure as the underlying kernel $K_{\kappa}$, according to definition (2.4) and Lemma 2.2. The structure of the solution to (2.1) with this special kernel can be found in [28]. The central goal of this paper is to analyze the structure of the solution to the equations for the case that the oscillatory component of the kernel is a nonlinear oscillator.

We next analyze the properties of the resolvent kernel associated with $K_{\kappa}$, where $K_{\kappa}$ belongs to the spaces introduced in [28]. We first recall the notions originally introduced in [28]. By an oscillatory function, we mean a function with numerous local maximum and minimum over the range of integration [7].

Definition 2.3. An oscillatory function $\varphi$ in a Banach space $\mathbb{X}$ with norm $\|\cdot\|_{\mathbb{X}}$ is called $\kappa$-oscillatory of order $n$ if $n$ is the smallest possible integer for which there exists a positive constant $c$ such that, for all $\kappa>1$ (especially for $\kappa \gg 1$ ),

$$
\kappa^{-n}\|\varphi\|_{\mathbb{X}} \leq c
$$

If $\varphi$ satisfies (2.7) with $n=0$, we say that $\varphi$ is non- $\kappa$-oscillatory. 
Definition 2.4. A subset

$$
\mathbb{X}_{\kappa, n}:=\{u \in \mathbb{X}: u \text { is } \kappa \text {-oscillatory of order } n\}
$$

of a normed space $\mathbb{X}$ is called a $\kappa$-oscillatory space of order $n$. If $n=0$, $\mathbb{X}_{\kappa, n}$ is called a non- $\kappa$-oscillatory space.

We now describe the spaces to be used in the remaining parts of the paper. For a non-negative integer $m$, let $C^{m}(I)$ denote the space of continuous functions with the norm

$$
\|L\|_{m}:=\max _{p \in \mathbb{Z}_{m}}\left\{\left\|L^{(p)}\right\|_{\infty}\right\}
$$

and let $C^{m}(D)$ denote the space (containing the functions that have continuous mixed partial derivatives of order $m$ with respect to $x$ and $y$ for $(x, y) \in D)$ with norm

$$
\|L\|_{m}:=\max \left\{\left\|L^{(p, q)}\right\|_{\infty}: p, q \in \mathbb{Z}_{m}, p+q \leq m\right\}
$$

where $L^{(p, q)}(x, y):=\mathcal{D}_{x}^{p} \mathcal{D}_{y}^{q} L(x, y)$ for $(x, y) \in D$. Note that, although the definition of $\|L\|_{m}$ in $C^{m}(I)$ is different from that in $C^{m}(D)$, the reader should be able to distinguish them from the context. We denote by $C_{\kappa, n}^{m}(I)$ the $\kappa$-oscillatory space of order $n \in \mathbb{Z}_{m}$ of the space $C^{m}(I)$, and by $C_{\kappa, n}^{m}(D)$ the $\kappa$-oscillatory space of order $n \in \mathbb{Z}_{m}$ of the space $C^{m}(D)$. Let

$$
C_{\kappa}^{m}(I):=\bigcap_{n \in \mathbb{Z}_{m}} C_{\kappa, n}^{n}(I), \quad \text { and } \quad C_{\kappa}^{m}(D):=\bigcap_{n \in \mathbb{Z}_{m}} C_{\kappa, n}^{n}(D) .
$$

We shall prove that, if $K_{\kappa} \in C_{\kappa}^{m}(D)$ and $f \in C_{\kappa}^{m}(I)$, then the solution $u$ of (2.1) belongs to the space $C_{\kappa}^{m}(I)$.

Next, we first analyze the iterated kernels $H_{n}$ for $n \in \mathbb{N}$ defined in (2.3). For this purpose, we recall Leibniz's formula for differentiating functions of the form

$$
\phi(x):=\int_{a(x)}^{b(x)} \varphi(x, y) \mathrm{d} y
$$

with respect to $x$, assuming that $\varphi, a$ and $b$ are smooth functions 
satisfying $a(x) \leq y \leq b(x)$. The formula has the form

$$
\phi^{\prime}(x)=\int_{a(x)}^{b(x)} \varphi_{x}(x, y) \mathrm{d} y+\varphi(x, b(x)) b^{\prime}(x)-\varphi(x, a(x)) a^{\prime}(x) .
$$

We also need the formula for a function $L \in C^{n}(D)$ with $n \in \mathbb{N}_{0}$,

$$
(L(t, t))^{(n)}=\sum_{j \in \mathbb{Z}_{n}} C_{n}^{j} L^{(j, n-j)}(t, t), \quad \text { for } t \in I,
$$

where $C_{n}^{j}:=n ! /(j !(n-j) !)$ for $n \in \mathbb{N}_{0}$ and $j \in \mathbb{Z}_{n}$ are the binomial coefficients.

Lemma 2.5. If $n \in \mathbb{N}$ with $n>1$ and $K_{\kappa} \in C^{n-2}(D)$, then for $j \in \mathbb{Z}_{n-2}$ and $i \in \mathbb{Z}_{j}$,

$$
H_{n}^{(i, j-i)}(t, t)=0, \quad \text { for } t \in I .
$$

Proof. We prove this result by induction on $n$. For $n=2$, the result follows directly from the definition (2.3) of $\mathrm{H}_{2}$. Assuming that this result is true for $n \in \mathbb{N}$ with $n>1$, consider the case $n+1$. According to the formula (2.8), we have that, for $j \in \mathbb{Z}_{n-1}, i \in \mathbb{Z}_{j}$ and $(t, s) \in D$,

$$
\begin{aligned}
H_{n+1}^{(i, j-i)}(t, s ; \kappa)= & \int_{s}^{t} H_{1}^{(i, 0)}(t, v ; \kappa) H_{n}^{(0, j-i)}(v, s ; \kappa) \mathrm{d} v \\
& +\sum_{l \in \mathbb{Z}_{i-1}}\left[H_{1}^{(l, 0)}(t, t ; \kappa) H_{n}(t, s ; \kappa)\right]^{(i-l-1, j-i)} \\
& -\sum_{k \in \mathbb{Z}_{j-i-1}}\left[H_{1}(t, s ; \kappa) H_{n}^{(0, k)}(s, s ; \kappa)\right]^{(i, j-i-k-1)} .
\end{aligned}
$$

It follows from formula (2.9) that, for $j \in \mathbb{Z}_{n-1}, i \in \mathbb{Z}_{j}, l \in \mathbb{Z}_{i-1}$, $k \in \mathbb{Z}_{j-i-1}$ and $(t, s) \in D$,

$$
\begin{aligned}
& {\left[H_{1}^{(l, 0)}(t, t ; \kappa) H_{n}(t, s ; \kappa)\right]^{(i-l-1, j-i)}} \\
& \quad=\sum_{p \in \mathbb{Z}_{i-l-1}} \sum_{q \in \mathbb{Z}_{p}} C_{i-l-1}^{p} C_{p}^{q} H_{1}^{(l+q, p-q)}(t, t ; \kappa) H_{n}^{(i-l-p-1, j-i)}(t, s ; \kappa)
\end{aligned}
$$

and 


$$
\begin{gathered}
{\left[H_{1}(t, s ; \kappa) H_{n}^{(0, k)}(s, s ; \kappa)\right]^{(i, j-i-k-1)}=\sum_{p \in \mathbb{Z}_{j-i-k-1}} \sum_{q \in \mathbb{Z}_{p}}} \\
C_{j-i-k-1}^{p} C_{p}^{q} H_{1}^{(i, j-i-k-p-1)}(t, s ; \kappa) H_{n}^{(q, p-q+k)}(s, s ; \kappa) .
\end{gathered}
$$

Applying equations (2.10) for $n$ to the equations (2.11), and using the two equations above, we obtain the conclusion for $n+1$. This completes the proof.

In the following lemmas, we derive a bound for the derivatives of $H_{n}, n \in \mathbb{N}$. In order to do so we note that, according to Definition 2.4, if $K_{\kappa} \in C_{\kappa}^{m}(D)$ for a fixed integer $m \in \mathbb{N}$, there exists a positive constant $c$ such that, for all $\kappa>1$ and $n \in \mathbb{Z}_{m}$,

$$
\left\|K_{\kappa}\right\|_{n} \leq c \kappa^{n} \text {. }
$$

For fixed $m \in \mathbb{N}$, we divide $H_{n}$ into two parts to estimate the bound. The first part is composed of $H_{n}$ for $n \in \mathbb{Z}_{2 m+3}$. The number of functions in this part is finite. We shall calculate the upper bound for the derivatives of functions in the first part directly. Then the number of functions in the other parts is infinite. To obtain an upper bound for the derivatives of the functions in the second part we use the properties of $H_{n}$ shown in Lemma 2.5.

Lemma 2.6. If $K_{\kappa} \in C_{\kappa}^{m}(D)$ for a fixed integer $m \in \mathbb{N}$, then there exists a positive constant $c$ such that, for all $\kappa>1, n \in \mathbb{Z}_{2 m+3}^{+}$and $j \in \mathbb{Z}_{m}$,

$$
\left\|H_{n}\right\|_{j} \leq c \kappa^{j}
$$

Proof. We proceed by induction on $n \in \mathbb{Z}_{2 m+3}^{+}$. The result for $n=1$ follows directly from equation (2.12) of $H_{1}$. Assume that this result is true for $n \in \mathbb{Z}_{2 m+2}^{+}$, and consider the case $n+1$. Note that there exists a positive constant $c$ such that, for all $\kappa>1, j \in \mathbb{Z}_{m}, i \in \mathbb{Z}_{j}, l \in \mathbb{Z}_{i-1}$ and $k \in \mathbb{Z}_{j-i-1}$

$$
\sum_{l \in \mathbb{Z}_{i-1}} \sum_{p \in \mathbb{Z}_{i-l-1}} \sum_{q \in \mathbb{Z}_{p}} C_{i-l-1}^{p} C_{p}^{q} \leq c
$$

and

$$
\sum_{k \in \mathbb{Z}_{j-i-1}} \sum_{p \in \mathbb{Z}_{j-i-k-1}} \sum_{q \in \mathbb{Z}_{p}} C_{j-i-k-1}^{p} C_{p}^{q} \leq c .
$$


Applying equation (2.11) with the assumption for $n$, we obtain that there exists a positive constant $c$ independent of $\kappa$ such that, for $j \in \mathbb{Z}_{m}$, $\left\|H_{n+1}\right\|_{j} \leq c \kappa^{j}$. The conclusion for $n+1$ now follows, thus completing the proof.

Lemma 2.7. If $K_{\kappa} \in C_{\kappa}^{m}(D)$ for a fixed integer $m \in \mathbb{N}$, then there exists a positive constant $c$ such that, for all $\kappa>1, n \in \mathbb{N}$ with $n \geq 2 m+4, j \in \mathbb{Z}_{m}$ and $i \in \mathbb{Z}_{j}$,

$$
\max _{(t, s) \in D}\left\{\left|H_{n}^{(i, j-i)}(t, s ; \kappa)\right|\right\} \leq c^{\lfloor n /(m+2)\rfloor} \frac{(t-s)^{\lfloor n /(m+2)\rfloor-1}}{(\lfloor n /(m+2)\rfloor-1) !} \kappa^{j},
$$

where $\lfloor a\rfloor$ denotes the biggest integer not exceeding $a$.

Proof. We employ induction on $l:=\lfloor n /(m+2)\rfloor$ for $n \geq 2 m+4$ satisfying $n=l(m+2)+k$ with $k \in \mathbb{Z}_{m+1}$. According to [2], we rewrite the iterated kernels in the form

$$
H_{l(m+2)+k}(t, s ; \kappa)=\int_{s}^{t} H_{m+2}(t, v ; \kappa) H_{(l-1)(m+2)+k}(v, s ; \kappa) \mathrm{d} v .
$$

Applying Lemma 2.5, we have, for $n \geq 2 m+4, j \in \mathbb{Z}_{m}$ and $i \in \mathbb{Z}_{j}$ that

$$
H_{l(m+2)+k}^{(i, j-i)}(t, s ; \kappa)=\int_{s}^{t} H_{m+2}^{(i, 0)}(t, v ; \kappa) H_{(l-1)(m+2)+k}^{(0, j-i)}(v, s ; \kappa) \mathrm{d} v .
$$

This, together with Lemma 2.6, yields the results for $l=2$ and $k \in \mathbb{Z}_{m+1}$. We assume that this result is true for $n=l(m+2)+k$ with $l \geq 2$ and $k \in \mathbb{Z}_{m+1}$, and consider the case $l+1$. Applying equation (2.14) with the assumption for $l$, we may obtain the conclusion for $l+1$. This finishes the proof.

We now present the two main results of this section. The first theorem describes the property of the resolvent kernel.

Theorem 2.8. If $K_{\kappa} \in C_{\kappa}^{m}(D)$ for a fixed integer $m \in \mathbb{N}$, then $R_{\kappa} \in C_{\kappa}^{m}(D)$, where $R_{\kappa}$ is the resolvent kernel associated with the given kernel $K_{\kappa}$ of (2.1).

Proof. Recalling Lemmas 2.6, 2.7 and definition (2.4) of the resolvent kernel $R_{\kappa}$ yields the conclusion, since the infinite sum of the iterated 
kernels (i.e., the Neumann series) converges absolutely and uniformly in $D$.

The second theorem shows the degree of oscillation of the solution to the VIE (2.1).

Theorem 2.9. If $f \in C_{\kappa}^{m}(I)$ and $K_{\kappa} \in C_{\kappa}^{m}(D)$ for a fixed integer $m \in \mathbb{N}$, then $u \in C_{\kappa}^{m}(I)$, where $u$ is the solution of equation (2.1).

Proof. We prove this result by applying Theorem 2.8 with the representation of $u$ by means of the resolvent kernel $R_{\kappa}$ and $f$. Differentiating equation (2.5) directly yields, for $j \in \mathbb{Z}_{m}$ and $t \in I$, that

$u^{(j)}(t)=f^{(j)}(t)+\int_{0}^{t} R_{\kappa}^{(j, 0)}(t, s) f(s) \mathrm{d} s+\sum_{i \in \mathbb{Z}_{j-1}}\left(R_{\kappa}^{(i, 0)}(t, t) f(t)\right)^{(j-i-1)}$.

Applying the formula (2.9), we have for $j \in \mathbb{Z}_{m}, i \in \mathbb{Z}_{j-1}$ and $t \in I$ that

$$
\begin{aligned}
& \left(R_{\kappa}^{(i, 0)}(t, t) f(t)\right)^{(j-i-1)} \\
& \quad=\sum_{p \in \mathbb{Z}_{j-i-1}} \sum_{q \in \mathbb{Z}_{p}} C_{j-i-1}^{p} C_{p}^{q} R_{\kappa}^{(i+q, p-q)}(t, t) f^{(j-i-p-1)}(t) .
\end{aligned}
$$

This, together with Theorem 2.8 and the assumption on $f$, leads to the desired result.

\section{The oscillation structure of the solution to the oscillatory}

VIEs. We study in this section the oscillation structure of the solution of VIEs (2.1) for the given kernel $K_{\kappa}$ with the oscillatory component $\mathrm{e}^{\mathrm{i} \kappa g}$, where $g$ is a nonlinear function in $D$. The idea of representing the solution of the VIEs by successive substitutions of the equation used in [28] is employed to analyze the properties of the solution, and the technique of changing the interval of integration into paths in the complex plane is used to decompose the oscillatory Volterra integral operators.

We begin by recalling the oscillatory structured spaces introduced originally in [28]. 
Definition 3.1. Let $\mathbb{X}$ be a normed space, and assume that $\varepsilon_{N}:=$ $\left\{e_{j} \in C(\Omega): j \in \mathbb{Z}_{N}^{+}\right\}$for $N \in \mathbb{N}$ is a set of oscillatory functions with the wave number $\kappa$. Moreover, suppose that $e_{j}$ with $j \in \mathbb{Z}_{N}^{+}$and 1 are linearly independent. A $\kappa$-oscillatory structured space of order $n$ with $\varepsilon_{N}$ is defined by

$$
\widetilde{\mathbb{X}}_{\kappa, n}:=\left\{u_{0}+\sum_{j \in \mathbb{Z}_{N}^{+}} u_{j} e_{j}: u_{j} \in \mathbb{X}_{\kappa, n}, j \in \mathbb{Z}_{N}\right\},
$$

equipped with the norm

$$
\|u\|_{\tilde{\mathbb{X}}_{\kappa, n}}:=\left(\sum_{j \in \mathbb{Z}_{N}}\left\|u_{j}\right\|_{\mathbb{X}}^{2}\right)^{1 / 2} .
$$

We first define the spaces to be used in this section. For a given oscillator $g \in C(D)$, we define

$$
\widetilde{e}_{1}(t, s):=\mathrm{e}^{\mathrm{i} \kappa g(t, s)}, \quad(t, s) \in D, \quad \text { and } \quad e_{1}(t):=\mathrm{e}^{\mathrm{i} \kappa g(t, 0)}, \quad t \in I .
$$

Correspondingly, we construct the $\kappa$-oscillatory structured spaces $\widetilde{C}_{\kappa, n}^{m}$ $(D)$ of order $n \in \mathbb{Z}_{m}$ with the structure $\left\{\widetilde{e}_{1}\right\}$ and the $\kappa$-oscillatory structured spaces $\widetilde{C}_{\kappa, n}^{m}(I)$ of order $n \in \mathbb{Z}_{m}$ with the structure $\left\{e_{1}\right\}$.

We shall prove that the solution $u$ of the VIEs (2.1) belongs to the space $\widetilde{C}_{\kappa, 0}^{m}(I)$, under the hypotheses that $K_{\kappa} \in \widetilde{C}_{\kappa, 0}^{m}(D)$ and $f \in$ $\widetilde{C}_{\kappa, 0}^{m}(I)$, complemented by appropriate assumptions on the oscillators $g, K_{\kappa}$ and $f$. Recalling (2.1), the solution $u$ satisfies the equation $u=f+\mathcal{V}_{\kappa} u$. By successive substitutions, we find that

$$
u=\mathcal{V}_{\kappa}^{n} u+\sum_{j \in \mathbb{Z}_{n-1}} \mathcal{V}_{\kappa}^{j} f
$$

The main work of this section is to prove that $\mathcal{V}_{\kappa}^{n} f \in \widetilde{C}_{\kappa, 0}^{m}(I)$ for $n \in \mathbb{N}$, and there exists a number $n \in \mathbb{N}$ such that $\mathcal{V}_{\kappa}^{n} \phi \in \widetilde{C}_{\kappa, 0}^{m}(I)$ for $\phi \in C_{\kappa}^{m}(I)$. We then obtain the form of $u$ from the equation (3.1) with the help of Theorem 2.9.

We now focus on analyzing the properties of $\mathcal{V}_{\kappa}^{n}$ for $n \in \mathbb{N}$ defined as in (2.2). For this purpose, we review a decomposition of the oscillatory integrals $[\mathbf{1 1}, \mathbf{1 2}]$. We first present the hypotheses on the integrand. 
Assumption 3.2. $\psi \in C^{m+1}(I)$ for some $m \in \mathbb{N}$, which is independent of $\kappa$, is analytic in a simply connected and infinite complex region $\Omega$ containing the interval $I$, and the inverse of $\psi$ exists on $\Omega$ with $\psi^{\prime}(z) \neq 0$ for $z \in \Omega$. Moreover, $\psi^{-1}(\psi(z)+\mathrm{i} \tau) \in \Omega$ for $z \in \Omega$ and $\tau \in(-\infty, \infty)$, and there exist constants $\gamma_{1, j} \geq 0$ and $\gamma_{2, j} \geq 0$ for $j \in \mathbb{Z}_{m+1}$, such that, for $z \in \Omega$,

$$
\left|\psi^{(j)}(z)\right|=\mathcal{O}\left(|z|^{\gamma_{1, j}}\right) \quad \text { and } \quad\left|\left(\psi^{-1}\right)^{(j)}(z)\right|=\mathcal{O}\left(|z|^{\gamma_{2, j}}\right) \text { as }|z| \rightarrow \infty \text {. }
$$

Assumption 3.3. $\phi \in C^{m}(I)$ for some $m \in \mathbb{N}$, which is independent of $\kappa$, is analytic in a simply connected and infinite complex region $\Omega$ containing the interval $I$, and there exist constants $\gamma_{j} \geq 0$ for $j \in \mathbb{Z}_{m}$, such that, for $z \in \Omega$,

$$
\left|\phi^{(j)}(z)\right|=\mathcal{O}\left(|z|^{\gamma_{j}}\right) \quad \text { as } \quad|z| \rightarrow \infty
$$

If $\psi$ and $\phi$ are analytic in a simply connected and infinite complex region $\Omega$ containing the interval $I$, they satisfy Assumption 3.2 and Assumption 3.3, respectively. For $t \in I$, we have

$$
\begin{gathered}
\int_{0}^{t} \phi(\tau) \mathrm{e}^{-\mathrm{i} \kappa \psi(\tau)} \mathrm{d} \tau=F_{-}(t)-F_{-}(0), \\
\int_{0}^{t} \phi(\tau) \mathrm{e}^{\mathrm{i} \kappa \psi(\tau)} \mathrm{d} \tau=F_{+}(0)-F_{+}(t),
\end{gathered}
$$

where the paths for $t \in I$ are defined by $h_{t}(\tau):=\psi^{-1}(\psi(t)+\mathrm{i} \tau)$ with $\tau \in(-\infty, \infty)$, and

$$
\begin{aligned}
& F_{-}(t):=\int_{-\infty}^{0} \phi\left(h_{t}(\tau)\right) \mathrm{e}^{-\mathrm{i} \kappa \psi\left(h_{t}(\tau)\right)} h_{t}^{\prime}(\tau) \mathrm{d} \tau, \\
& F_{+}(t):=\int_{0}^{\infty} \phi\left(h_{t}(\tau)\right) \mathrm{e}^{\mathrm{i} \kappa \psi\left(h_{t}(\tau)\right)} h_{t}^{\prime}(\tau) \mathrm{d} \tau .
\end{aligned}
$$

Since we utilize the above method to decompose the oscillatory integrals, we shall add the analyticity to the kernels $K_{\kappa}$ and $f$. We therefore present these assumptions as follows.

Assumption 3.4. $L \in C^{m}(D)$ for some $m \in \mathbb{N}$, which is independent of $\kappa$, is analytic in a simply connected and infinite complex region $\Omega^{2}$ 
with $\Omega$ containing the interval $I$, and there exist constants $\gamma_{i, j}^{1} \geq 0$ and $\gamma_{i, j}^{2} \geq 0$ for $j \in \mathbb{Z}_{m}$ and $i \in \mathbb{Z}_{j}$, such that, for $\left(z_{1}, z_{2}\right) \in \Omega^{2}$,

$$
\left|L^{(i, j-i)}\left(z_{1}, z_{2}\right)\right|=\mathcal{O}\left(\left|z_{1}\right|^{\gamma_{i, j}^{1}}+\left|z_{2}\right|^{\gamma_{i, j}^{2}}\right) \quad \text { as } \quad\left|z_{1}\right|,\left|z_{2}\right| \rightarrow \infty .
$$

Assumption 3.5. The function $g$ has the form $g(t, s):=g_{0}(t)-g_{0}(s)$ for $(t, s) \in D$, where the function $g_{0} \in C^{m+1}(I)$ for some $m \in \mathbb{N}$ is strictly increasing and satisfies Assumption 3.2.

Assumption 3.6. The kernel $K_{\kappa} \in \widetilde{C}_{\kappa, 0}^{m}(D)$ for some $m \in \mathbb{N}$ has the form $K_{\kappa}:=L_{1} \widetilde{e}_{1}+L_{0}$, where $L_{0}, L_{1} \in C_{\kappa, 0}^{m}(D)$ satisfy Assumption 3.4 .

Assumption 3.7. The function $f \in \widetilde{C}_{\kappa, 0}^{m}(I)$ for some $m \in \mathbb{N}$ has the form $f:=f_{1} e_{1}+f_{0}$, where $f_{0}, f_{1} \in C_{\kappa, 0}^{m}(I)$ satisfy Assumption 3.3.

In passing, we comment on the hypothesis imposed on $g$ and $\Omega$. If $g(t, s)=t-s$ for $(t, s) \in D, g$ satisfies Assumption 3.5 with $g_{0}(t)=t$ for $t \in I$. $\Omega$ is the intersection of the analytic complex regions of $f, g$ and $K_{\kappa}$.

We next decompose a class of Volterra integral functions defined by

$$
\widetilde{L}_{ \pm}(t ; \kappa):=\int_{0}^{t} L(t, s) \mathrm{e}^{ \pm \mathrm{i} \kappa g_{0}(s)} \mathrm{d} s, \quad \text { for } t \in I,
$$

where $g_{0} \in C^{m+1}(I)$ is strictly increasing and satisfies Assumption 3.2 for some $m \in \mathbb{N}$, and $L \in C_{\kappa, 0}^{m}(D)$ satisfies Assumption 3.4. According to $(3.2)$, we define two functions for $(t, s) \in D$ by

$$
\begin{aligned}
& F_{-}(t, s):=\int_{-\infty}^{0} L(t, h(s, \tau)) h^{(0,1)}(s, \tau) \mathrm{e}^{\kappa \tau} \mathrm{d} \tau, \\
& F_{+}(t, s):=\int_{0}^{\infty} L(t, h(s, \tau)) h^{(0,1)}(s, \tau) \mathrm{e}^{-\kappa \tau} \mathrm{d} \tau,
\end{aligned}
$$

where

$$
h(s, \tau):=g_{0}^{-1}\left(g_{0}(s)+\mathrm{i} \tau\right), \quad \text { for } s \in I \text { and } \tau \in(-\infty, \infty) .
$$

The functions defined by (3.3) have the decomposition

$$
\widetilde{L}_{ \pm}(t ; \kappa)= \pm F_{ \pm}(t, 0) \mathrm{e}^{ \pm \mathrm{i} \kappa g_{0}(0)} \mp F_{ \pm}(t, t) \mathrm{e}^{ \pm \mathrm{i} \kappa g_{0}(t)}, \quad \text { for } t \in I .
$$


We analyze the properties of $h$ defined by (3.4) in the following lemma. This requires the use of the Faà di Bruno formula $[\mathbf{1 5}, \mathbf{2 3}, \mathbf{2 4}]$ for derivatives of the composition of two functions. For a fixed $n \in \mathbb{N}$, if the derivatives of order $n$ of two functions $\phi$ and $\psi$ are defined, then

$$
(\phi \circ \psi)^{(n)}=\sum_{j \in \mathbb{Z}_{n}^{+}} \phi^{(j)}(\psi) B_{n, j}\left(\psi^{(1)}, \psi^{(2)}, \ldots, \psi^{(n-j+1)}\right),
$$

where, for $j \in \mathbb{Z}_{n}^{+}$,

$$
B_{n, j}\left(x_{1}, x_{2}, \ldots, x_{n-j+1}\right)=\sum \frac{n !}{m_{1} ! m_{2} ! \cdots m_{n-j+1} !} \prod_{l \in \mathbb{Z}_{n-j+1}^{+}}\left(\frac{x_{l}}{l !}\right)^{m_{l}}
$$

where the sum is taken over all $(n-j+1)$-tuples $\left(m_{1}, \ldots, m_{n-j+1}\right)$ satisfying the constraints

$$
\sum_{l \in \mathbb{Z}_{n-j+1}^{+}} m_{l}=j \text { and } \sum_{l \in \mathbb{Z}_{n-j+1}^{+}} l m_{l}=n .
$$

Lemma 3.8. If $g_{0} \in C^{m+1}(I)$ for some $m \in \mathbb{N}$ is strictly increasing and satisfies Assumption 3.2, then, for fixed $\tau \in(-\infty, \infty), h(\cdot, \tau)$ defined by $(3.4)$ and $h^{(0,1)}(\cdot, \tau)$ satisfy Assumption 3.3 .

Proof. We first verify the assertion for $h$. From definition (3.4), we have that, for $z \in \Omega, h(z, \tau)=g_{0}^{-1}\left(g_{0}(z)+\mathrm{i} \tau\right)$. According to the assumptions on $g_{0}$, there exists a constant $\gamma_{1,0} \geq 0$ such that $|h(z, \tau)|=\left|g_{0}^{-1}\left(\mathcal{O}\left(|z|^{\gamma_{1,0}}\right)+\mathrm{i} \tau\right)\right|$ as $|z| \rightarrow \infty$. We then obtain that there exists a constant $\gamma_{0} \geq 0$ such that $|h(z, \tau)|=\mathcal{O}\left(|z|^{\gamma_{0}}\right)$ as $|z| \rightarrow \infty$. For $n \in \mathbb{Z}_{m}^{+}$, using formula (3.6) yields that, for $z \in \Omega$,

$$
\begin{aligned}
& h^{(n, 0)}(z, \tau) \\
& =\sum_{j \in \mathbb{Z}_{n}^{+}}\left(g_{0}^{-1}\right)^{(j)}\left(g_{0}(z)+\mathrm{i} \tau\right) B_{n, j}\left(g_{0}^{(1)}(z), g_{0}^{(2)}(z), \ldots, g_{0}^{(n-j+1)}(z)\right) .
\end{aligned}
$$

Applying Assumption 3.2 to $g_{0}$ we obtain that there exists a constant $\gamma_{n} \geq 0$ such that $\left|h^{(n, 0)}(z, \tau)\right|=\mathcal{O}\left(|z|^{\gamma_{n}}\right)$ as $|z| \rightarrow \infty$. We can obtain the result for $h^{(0,1)}$ by the same method used above. The proof is complete. 
The next theorem states the properties of $\mathcal{V}_{\kappa}^{n} f$ for $n \in \mathbb{N}$ and $f \in \widetilde{C}_{\kappa, 0}^{m}(I)$. For this purpose, we assume throughout the subsequent parts of the paper that $\Omega$ has the following property: for any $t \in I$ and $z \in \Omega$, the straight line connecting $t$ and $z$ lies wholly within $\Omega$.

Theorem 3.9. If $g \in C^{m+1}(D)$ for some $m \in \mathbb{N}$ satisfies Assumption 3.5 and $K_{\kappa} \in \widetilde{C}_{\kappa, 0}^{m}(D)$ satisfies Assumption 3.6, then $f \in \tilde{C}_{\kappa, 0}^{m}(I)$ satisfies Assumption 3.7, $\mathcal{V}_{\kappa}^{n} f \in \widetilde{C}_{\kappa, 0}^{m}(I)$ for $n \in \mathbb{N}$.

Proof. We prove this result by induction on $n$. We first analyze $\mathcal{V}_{\kappa} f$. Defining $w_{1}(t):=\left(\mathcal{V}_{\kappa} w_{0}\right)(t)$ for $t \in I$ with $w_{0}:=f$, we see that, for $t \in I$,

$$
\begin{aligned}
w_{1}(t)= & e_{1}(t) \alpha_{0}(t)+\beta_{0}(t) \\
& +\int_{0}^{t} L_{1}(t, s) f_{0}(s) \mathrm{e}^{\mathrm{i} \kappa g(t, s)} \mathrm{d} s+\int_{0}^{t} L_{0}(t, s) f_{1}(s) \mathrm{e}^{\mathrm{i} \kappa g(s, 0)} \mathrm{d} s,
\end{aligned}
$$

where, for $t \in I$,

$$
\alpha_{0}(t):=\int_{0}^{t} L_{1}(t, s) f_{1}(s) \mathrm{d} s
$$

and

$$
\beta_{0}(t):=\int_{0}^{t} L_{0}(t, s) f_{0}(s) \mathrm{d} s .
$$

By replacing $L$ by $L_{1} f_{0}$ and $L_{0} f_{1}$ in (3.3), we obtain for $t \in I$ that

$$
\begin{aligned}
& \int_{0}^{t} L_{1}(t, s) f_{0}(s) \mathrm{e}^{\mathrm{i} \kappa g(t, s)} \mathrm{d} s=-e_{1}(t) \alpha_{1}(t)+\beta_{1}(t), \\
& \int_{0}^{t} L_{0}(t, s) f_{1}(s) \mathrm{e}^{\mathrm{i} \kappa g(s, 0)} \mathrm{d} s=-e_{1}(t) \alpha_{2}(t)+\beta_{2}(t),
\end{aligned}
$$

where $\alpha_{1}(t):=F_{-}(t, 0), \beta_{1}(t):=F_{-}(t, t), \alpha_{2}(t):=F_{+}(t, t)$ and $\beta_{2}(t):=F_{+}(t, 0)$ for $t \in I$. Note that the functions $\alpha_{j}$ and $\beta_{j}$ for $j \in \mathbb{Z}_{2}$ are independent of $\kappa$. We therefore obtain that $w_{1} \in \widetilde{C}_{\kappa, 0}^{m}(I)$.

To prove the conclusion by induction we next need to verify that $\alpha_{j}$ and $\beta_{j}$ for $j \in \mathbb{Z}_{2}$ satisfy Assumption 3.3. We first consider $\alpha_{0}$. Using 
formula (2.8), we have that, for $z \in \Omega$ and $j \in \mathbb{Z}_{m}$,

$$
\alpha_{0}^{(j)}(z)=\int_{0}^{z} L_{1}^{(j, 0)}(z, s) f_{1}(s) \mathrm{d} s+\sum_{i \in \mathbb{Z}_{j-1}}\left[L_{1}^{(i, 0)}(z, z) f_{1}(z)\right]^{(j-i-1)} .
$$

Let $\iota$ denote the straight line that connects 0 and $z$ in $\Omega$. We find that the upper bound of the first term in the right hand side of the equation above is

$$
\max _{s \in \iota}\left\{\left|L_{1}^{(j, 0)}(z, s) f_{1}(s)\right||z|\right\} .
$$

This, together with the assumptions on $L_{1}$ and $f_{1}$, yields that $\alpha_{0}$ satisfies Assumption 3.3. We then analyze $\alpha_{1}$ where, for $z \in \Omega$ and $j \in \mathbb{Z}_{m}$,

$$
\alpha_{1}^{(j)}(z)=\int_{-\infty}^{0} L_{1}^{(j, 0)}(z, h(0, \tau)) f_{0}(h(0, \tau)) h^{(0,1)}(0, \tau) \mathrm{e}^{\kappa \tau} \mathrm{d} \tau .
$$

Applying the assumptions on $L_{1}$ and $f_{0}$ yields that $\alpha_{1}$ satisfies Assumption 3.3. We next consider $\alpha_{2}$. From the definition of $F_{+}$, we have that, for $z \in \Omega$ and $j \in \mathbb{Z}_{m}$,

$$
\alpha_{2}^{(j)}(z)=\int_{0}^{\infty} \mathcal{D}_{z}^{j}\left[L_{0}(z, h(z, \tau)) f_{1}(h(z, \tau)) h^{(0,1)}(z, \tau)\right] \mathrm{e}^{-\kappa \tau} \mathrm{d} \tau .
$$

Applying the assumptions on $g, L_{0}$ and $f_{1}$ with Lemma 3.8 , we obtain that $\alpha_{2}$ satisfies Assumption 3.3. By using the same method we get that $\beta_{j}$ for $j \in \mathbb{Z}_{2}$ satisfies Assumption 3.3. This yields that $w_{1} \in \widetilde{C}_{\kappa, 0}^{m}(I)$ satisfies Assumption 3.7.

We finally assume that this result is true for $n \in \mathbb{N}$, i.e., $w_{n}:=$ $\mathcal{V}_{\kappa}^{n} w_{n-1} \in \widetilde{C}_{\kappa, 0}^{m}(I)$ and consider the case $n+1$. We can obtain the result for $n+1$ by the same method used above. This finishes the proof.

We now establish for $K_{\kappa} \in \widetilde{C}_{\kappa, 0}^{m}(D)$ that the operator $\mathcal{V}_{\kappa}^{n}$ reduces the oscillatory order of functions in $C_{\kappa}^{m}(I)$ by $n \in \mathbb{N}$. To see this, we recall the definition of the less oscillatory kernel according to [28]. We say that $L \in C_{\kappa, 0}^{m}(D)$ is a less oscillatory kernel of order $n$ for $n \in \mathbb{Z}_{m}^{+}$ if there exists a positive constant $c$ such that, for all $\kappa>1, j \in \mathbb{Z}_{n-1}$ and $i \in \mathbb{Z}_{j}$,

$$
\max _{t \in I}\left\{\left|L^{(i, j-i)}(t, t)\right|\right\} \leq c \kappa^{-(n-j-1)} .
$$


For $L \in C_{\kappa, 0}^{m}(D)$, we define an operator on $C(I)$ by

$$
\left(\mathcal{W}_{\kappa}[L] \phi\right)(t):=\int_{0}^{t} L(t, s) \mathrm{e}^{\mathrm{i} \kappa g(t, s)} \phi(s) \mathrm{d} s, \quad \text { for } t \in I .
$$

Note that $\mathcal{W}_{0}[L]$ denotes a Volterra integral operator with nonoscillatory kernel. According to [28], if $L \in C_{\kappa, 0}^{m}(D)$ is a less oscillatory kernel of order $n$ for $n \in \mathbb{Z}_{m}^{+}$and $g(t, s)=t-s$ for $(t, s) \in D$, the operator $\mathcal{W}_{\kappa}[L]$ maps $C_{\kappa}^{m}(I)$ into $\widetilde{C}_{\kappa, m-n}^{m}(I)$. In the following lemma, we extend this property of $\mathcal{W}_{\kappa}[L]$ to the nonlinear oscillator $g$ satisfying Assumption 3.5.

Lemma 3.10. If $L \in C_{\kappa, 0}^{m}(D)$ for some $m \in \mathbb{N}$ is a less oscillatory kernel of order $n$ for $n \in \mathbb{Z}_{m}^{+}$and $g \in C^{m+1}(D)$ satisfies Assumption 3.5, then the operators $\mathcal{W}_{\kappa}[L]$ and $\mathcal{W}_{0}[L] \operatorname{map} C_{\kappa}^{m}(I)$ into $\widetilde{C}_{\kappa, m-n}^{m}(I)$.

Proof. For $\phi \in C_{\kappa}^{m}(I)$, we define $\left(\mathcal{W}_{\kappa}[L] \phi\right)(t)=w_{0}(t) \mathrm{e}^{\mathrm{i} \kappa g(t, 0)}$ and $w_{1}(t):=\left(\mathcal{W}_{0}[L] \phi\right)(t)$ for $t \in I$, where $w_{0}(t):=\int_{0}^{t} L(t, s) \mathrm{e}^{\mathrm{i} \kappa g(0, s)} \phi(s) \mathrm{d} s$. It suffices to prove that $w_{0}, w_{1} \in C_{\kappa, m-n}^{m}(I)$. We can use the method described in [28] to prove these results but omit the proof.

We first consider the case where $K_{\kappa} \in \widetilde{C}_{\kappa, 0}^{m}(D)$ satisfies Assumption 3.6 with the form $K_{\kappa}(t, s)=L_{1}(t, s) \mathrm{e}^{\mathrm{i} \kappa g(t, s)}$ for $(t, s) \in D$. For $n \in \mathbb{N}$ with $n>1$, let

$$
L_{n}(t, s):=\int_{s}^{t} L_{1}(t, v) L_{n-1}(v, s) \mathrm{d} v \quad \text { for }(t, s) \in D .
$$

We obtain that $\mathcal{V}_{\kappa}^{n}=\mathcal{W}_{\kappa}\left[L_{n}\right], n \in \mathbb{N}$. We show the property of $L_{n}$ in the following lemma.

Lemma 3.11. If $g \in C^{m+1}(D)$ satisfies Assumption 3.5 for some $m \in \mathbb{N}$ and $K_{\kappa} \in \widetilde{C}_{\kappa, 0}^{m}(D)$ satisfies Assumption 3.6 with $L_{0}=0$, then $L_{n} \in C_{\kappa, 0}^{m}(D)$ for $n \in \mathbb{Z}_{m}^{+}$is a less oscillatory kernel of order $n$.

Proof. We prove this result by induction on $n$. According to the assumption on $K_{\kappa}$, we obtain that $L_{1} \in C_{\kappa, 0}^{m}(D)$ is a less oscillatory kernel of order 1 . Assuming that this result is true for $n \in \mathbb{Z}_{m-1}^{+}$, we consider the case $n+1$. Using formula (2.8), we have that, for $j \in \mathbb{Z}_{n}$, 
$i \in \mathbb{Z}_{j}$ and $(t, s) \in D$,

$$
\begin{aligned}
L_{n+1}^{(i, j-i)}(t, s)= & \int_{s}^{t} L_{1}^{(i, 0)}(t, v) L_{n}^{(0, j-i)}(v, s) \mathrm{d} v \\
& +\sum_{l \in \mathbb{Z}_{i-1}}\left[L_{1}^{(l, 0)}(t, t) L_{n}(t, s)\right]^{(i-l-1, j-i)} \\
& -\sum_{k \in \mathbb{Z}_{j-i-1}}\left[L_{1}(t, s) L_{n}^{(0, k)}(s, s)\right]^{(i, j-i-k-1)} .
\end{aligned}
$$

This leads to the fact that there exists a positive constant $c$ such that, for all $\kappa>1, j \in \mathbb{Z}_{n}$ and $i \in \mathbb{Z}_{j}$,

$$
\max _{t \in I}\left\{\left|L_{n+1}^{(i, j-i)}(t, t)\right|\right\} \leq c \kappa^{-(n-j)} .
$$

We then obtain the conclusion of $n+1$. This completes the proof.

The structure of the solution for the case above is described in the following theorem.

Theorem 3.12. If $g \in C^{m+1}(D)$ satisfies Assumption 3.5 for some $m \in \mathbb{N}, K_{\kappa} \in \widetilde{C}_{\kappa, 0}^{m}(D)$ satisfies Assumption 3.6 with $L_{0}=0$, and $f \in \widetilde{C}_{\kappa, 0}^{m}(I)$ satisfies Assumption 3.7, then the solution $u$ of the VIE (2.1) belongs to the space $\widetilde{C}_{\kappa, 0}^{m}(I)$.

Proof. We prove this result by applying equation (3.1) for $n=m$. According to the assumptions on $K_{\kappa}$ and $g$, we have that $L_{m} \in C_{\kappa, 0}^{m}(D)$ is a less oscillatory kernel of order $m$. Note that $\mathcal{V}_{\kappa}^{m}=\mathcal{W}_{\kappa}\left[L_{m}\right]$. We obtain that $\mathcal{V}_{\kappa}^{m}$ maps $C_{\kappa}^{m}(I)$ into $\widetilde{C}_{\kappa, 0}^{m}(I)$. This, together with Theorems 2.9 and 3.9 , yields the conclusion.

We next assume that $K_{\kappa} \in \widetilde{C}_{\kappa, 0}^{m}(D)$ satisfying Assumption 3.6 has the form

$$
K_{\kappa}(t, s)=L_{0,1}(t, s) \mathrm{e}^{\mathrm{i} \kappa g(t, s)}+L_{0,0}(t, s) \quad \text { for }(t, s) \in D,
$$


with $L_{0,0}$ and $L_{0,1} \in C_{\kappa, 0}^{m}(D)$. According to Assumption 3.6, we have that $L_{0,0}=L_{0}$ and $L_{0,1}=L_{1}$. For $n \in \mathbb{N}$ and $(t, s) \in D$, we define

$$
\begin{aligned}
G_{n, 0}(t, s) & :=\int_{s}^{t} L_{0,0}(t, v) L_{n-1,0}(v, s) \mathrm{d} v \\
G_{n, 1}(t, s) & :=\int_{s}^{t} L_{0,1}(t, v) L_{n-1,1}(v, s) \mathrm{d} v \\
G_{n, 2}(t, s) & :=\int_{s}^{t} L_{0,0}(t, v) L_{n-1,1}(v, s) \mathrm{e}^{\mathrm{i} \kappa g(v, s)} \mathrm{d} v, \\
G_{n, 3}(t, s) & :=\int_{s}^{t} L_{0,1}(t, v) L_{n-1,0}(v, s) \mathrm{e}^{\mathrm{i} \kappa g(t, v)} \mathrm{d} v .
\end{aligned}
$$

To separate the oscillation of $G_{n, 2}$ and $G_{n, 3}$ as the function (3.3), we define, for $(t, s) \in D$,

$$
\begin{aligned}
R_{n, 0}(t, s) & :=\left(\mathcal{F}_{+}\left[L_{0,0} L_{n-1,1}\right]\right)(t, s, s), \\
R_{n, 1}(t, s) & :=\left(\mathcal{F}_{+}\left[L_{0,0} L_{n-1,1}\right]\right)(t, s, t), \\
J_{n, 0}(t, s) & :=\left(\mathcal{F}_{-}\left[L_{0,1} L_{n-1,0}\right]\right)(t, s, t), \\
J_{n, 1}(t, s) & :=\left(\mathcal{F}_{-}\left[L_{0,1} L_{n-1,0}\right]\right)(t, s, s),
\end{aligned}
$$

where, for an integrable function $L(t, s, v)$ with $0 \leq s \leq v \leq t \leq 1$,

$$
\begin{aligned}
& \left(\mathcal{F}_{+}[L]\right)(t, s, v):=\int_{0}^{\infty} L(t, s, h(v, \tau)) h^{(0,1)}(v, \tau) \mathrm{e}^{-\kappa \tau} \mathrm{d} \tau, \\
& \left(\mathcal{F}_{-}[L]\right)(t, s, v):=\int_{-\infty}^{0} L(t, s, h(v, \tau)) h^{(0,1)}(v, \tau) \mathrm{e}^{\kappa \tau} \mathrm{d} \tau .
\end{aligned}
$$

We obtain for $(t, s) \in D$ that

$$
G_{n, 2}(t, s)=-R_{n, 1}(t, s) \mathrm{e}^{\mathrm{i} \kappa g(t, s)}+R_{n, 0}(t, s)
$$

and

$$
G_{n, 3}(t, s)=-J_{n, 1}(t, s) \mathrm{e}^{\mathrm{i} \kappa g(t, s)}+J_{n, 0}(t, s) .
$$

For $n \in \mathbb{N}$, we then define

$$
L_{n, 0}(t, s):=G_{n, 0}(t, s)+R_{n, 0}(t, s)+J_{n, 0}(t, s)
$$

and

$$
L_{n, 1}(t, s):=G_{n, 1}(t, s)-R_{n, 1}(t, s)-J_{n, 1}(t, s) \quad \text { for }(t, s) \in D
$$


According to the definitions in (3.10) and (3.11), we obtain that the decomposition for the iterated integral operators of high orders is $\mathcal{V}_{\kappa}^{n}=\mathcal{W}_{\kappa}\left[L_{n-1,1}\right]+\mathcal{W}_{0}\left[L_{n-1,0}\right], n \in \mathbb{N}$. To analyze the properties of $\mathcal{V}_{\kappa}^{n}$, we verify that the functions $G_{n-1,0}, G_{n-1,1}, R_{n-1,0}, R_{n-1,1}$, $J_{n-1,0}$ and $J_{n-1,1}$ are less oscillatory kernels of order $n$ for $n \in \mathbb{Z}_{m}^{+}$ with $n>1$. According to the definitions (3.8) and (3.9), we introduce the following assumption.

Assumption 3.13. $L \in C^{m}(D)$ for some $m \in \mathbb{N}$ is independent of $\kappa$, and $L(\cdot, t)$ for $t \in I$ is analytic in a simply connected and infinite complex region $\Omega$ containing the interval $I$. In addition, there exist constants $\gamma_{i, j} \geq 0$ for $j \in \mathbb{Z}_{m}$ and $i \in \mathbb{Z}_{j}$ such that, for $z \in \Omega$ and $t \in I$,

$$
\left|L^{(i, j-i)}(z, t)\right|=\mathcal{O}\left(|z|^{\gamma_{i, j}}\right) \quad \text { as } \quad|z| \rightarrow \infty .
$$

We first show the property of $G_{n, 0}$ and $G_{n, 1}$ in the following lemma.

Lemma 3.14. If $g \in C^{m+1}(D)$ satisfies Assumption 3.5 for some $m \in \mathbb{N}, K_{\kappa} \in \widetilde{C}_{\kappa, 0}^{m}(D)$ satisfies Assumption 3.6, and $L_{n-1,0}, L_{n-1,1} \in$ $C_{\kappa, 0}^{m}(D)$ are less oscillatory kernels of order $n$ for $n \in \mathbb{Z}_{m-1}^{+}$which satisfy Assumption 3.13, then $G_{n, 0}, G_{n, 1} \in C_{\kappa, 0}^{m}(D)$ are less oscillatory kernels of order $n+1$ and satisfy Assumption 3.13.

Proof. The first result of this lemma can be proved as the one in Lemma 3.11. We focus on verifying the second result. Using the definition of $G_{n, 0}$ we have that, for $t \in I$ and $z \in \Omega$,

$$
G_{n, 0}(z, t)=\int_{t}^{z} L_{0,0}(z, v) L_{n-1,0}(v, t) \mathrm{d} v .
$$

Using formula (2.8), it follows for $j \in \mathbb{Z}_{m}, i \in \mathbb{Z}_{j}, t \in I$ and $z \in \Omega$ that

$$
\begin{aligned}
G_{n, 0}^{(i, j-i)}(z, t)= & \int_{t}^{z} L_{0,0}^{(i, 0)}(z, v) L_{n-1,0}^{(0, j-i)}(v, t) \mathrm{d} v \\
& +\sum_{l \in \mathbb{Z}_{i-1}}\left[L_{0,0}^{(l, 0)}(z, z) L_{n-1,0}(z, t)\right]^{(i-l-1, j-i)} \\
& -\sum_{k \in \mathbb{Z}_{j-i-1}}\left[L_{0,0}(z, t) L_{n-1,0}^{(0, k)}(t, t)\right]^{(i, j-i-k-1)} .
\end{aligned}
$$


According to the assumptions on $K_{\kappa}, L_{n-1,0}$ and $L_{n-1,1}$, we obtain that $G_{n, 0}$ satisfies Assumption 3.13. We can verify the assertion for $G_{n, 1}$ by the same method. This completes the proof.

We next analyze the properties of functions $R_{n, 0}, R_{n, 1}, J_{n, 0}$ and $J_{n, 1}$.

Lemma 3.15. If $g \in C^{2 m+1}(D)$ satisfies Assumption 3.5 for some $m \in \mathbb{N}, K_{\kappa} \in \widetilde{C}_{\kappa, 0}^{2 m}(D)$ satisfies Assumption 3.6, and $L_{n-1,0}, L_{n-1,1} \in$ $C_{\kappa, 0}^{2 m}(D)$ are less oscillatory kernels of order $n$ for $n \in \mathbb{Z}_{m-1}^{+}$which satisfy Assumption 3.13, then $R_{n, 0}, R_{n, 1}, J_{n, 0}$ and $J_{n, 1} \in C_{\kappa, 0}^{2 m}(D)$ are less oscillatory kernels of order $n+1$ and satisfy Assumption 3.13.

Proof. Using the definitions (3.8), (3.9) and the assumptions of this lemma, we can verify that $R_{n, 0}, R_{n, 1}, J_{n, 0}$ and $J_{n, 1} \in C_{\kappa, 0}^{2 m}(D)$. Note that $R_{n, 0}, R_{n, 1}, J_{n, 0}$ and $J_{n, 1}$ have a similar structure. We shall only verify that $R_{n, 0}$ is a less oscillatory kernel of order $n+1$ and satisfies Assumption 3.13. The analogous assertions for $R_{n, 1}, J_{n, 0}$ and $J_{n, 1}$ can be proved in the same way.

We first prove that $R_{n, 0}$ satisfies (3.7) with $n=n+1$ for $n \in \mathbb{Z}_{m-1}^{+}$. Note that $R_{n, 0}$ admits the explicit expression for $(t, s) \in D$

$$
R_{n, 0}(t, s)=\int_{0}^{\infty} L_{0,0}(t, h(s, \tau)) L_{n-1,1}(h(s, \tau), s) h^{(0,1)}(s, \tau) \mathrm{e}^{-\kappa \tau} \mathrm{d} \tau .
$$

For $(t, s) \in D$, we define

$$
\Theta(\tau ; t, s):=L_{0,0}(t, h(s, \tau)) L_{n-1,1}(h(s, \tau), s) h^{(0,1)}(s, \tau)
$$

for $\tau \in[0, \infty)$, which is a function with respect to $\tau$. By integration by parts and the assumptions on $g, L_{n-1,0}$ and $L_{n-1,1}$, we have that, for $(t, s) \in D$,

$$
R_{n, 0}(t, s)=\sum_{p \in \mathbb{Z}_{n-1}} \frac{\Theta^{(p)}(0 ; t, s)}{\kappa^{p+1}}+\frac{1}{\kappa^{n}} \int_{0}^{\infty} \Theta^{(n)}(\tau ; t, s) \mathrm{e}^{-\kappa \tau} \mathrm{d} \tau
$$

We now focus on estimating the bound of $\left(\Theta^{(p)}(0 ; t, s)\right)^{(i, j-i)}$ for $j \in \mathbb{Z}_{n}$ and $i \in \mathbb{Z}_{j}$. For this purpose, we first derive

$$
\eta_{p}(\tau, s):=\mathcal{D}_{\tau}^{p}\left(L_{n-1,1}(h(s, \tau), s)\right) \quad \text { for } p \in \mathbb{Z}_{n-1} .
$$


Applying formula (3.6), we see for $p \in \mathbb{Z}_{n-1}^{+}$that

$$
\begin{aligned}
\eta_{p}(\tau, s) & =\sum_{q \in \mathbb{Z}_{p}^{+}} L_{n-1,1}^{(q, 0)}(h(s, \tau), s) \\
& \times B_{p, q}\left((h(s, \tau))^{(0,1)},(h(s, \tau))^{(0,2)}, \ldots,(h(s, \tau))^{(0, p-q+1)}\right) .
\end{aligned}
$$

This leads to $\eta_{0}(0, s)=L_{n-1,1}(s, s)$ and

$$
\eta_{p}(0, s)=\sum_{q \in \mathbb{Z}_{p}^{+}} a_{p, q}(s) L_{n-1,1}^{(q, 0)}(s, s),
$$

where $a_{p, q}(s)$ is defined by

$$
B_{p, q}\left((h(s, \tau))^{(0,1)},(h(s, \tau))^{(0,2)}, \ldots,(h(s, \tau))^{(0, p-q+1)}\right)
$$

with $\tau=0$. We obtain that there exists a positive constant $c$ such that, for all $\kappa>1, j \in \mathbb{Z}_{n}$ and $p \in \mathbb{Z}_{n-1}$,

$$
\begin{aligned}
\frac{\left|\eta_{p}^{(j)}(0, s)\right|}{\kappa^{p+1}} \leq c \kappa^{-p-1} \kappa^{-(n-j-p-1)} \leq c \kappa^{-(n-j)} & \text { for } j<n-p, \\
& \frac{\left|\eta_{p}^{(j)}(0, s)\right|}{\kappa^{p+1}} \leq c \kappa^{-p-1} \leq c \kappa^{-(n-j)} \quad \text { for } j \geq n-p,
\end{aligned}
$$

since $p+1 \geq n-j+1>n-j$. This ensures that there exists a positive constant $c$ independent of $\kappa$ such that, for all $j \in \mathbb{Z}_{n}$ and $i \in \mathbb{Z}_{j}$,

$$
\left|\left(\Theta^{(p)}(0 ; s, s)\right)^{(i, j-i)}\right| \leq c \kappa^{n-j}, \quad \text { for } s \in I \text {. }
$$

Notice that the upper bound of the term with integral in the equation (3.12) is $c \kappa^{-n}$ for a positive constant $c$ independent of $\kappa$, since $L_{0,0} \in C_{\kappa, 0}^{2 m}(D)$ satisfies Assumption 3.4 and $L_{n-1,1} \in C_{\kappa, 0}^{2 m}(D)$ satisfies Assumption 3.13. We then obtain that $R_{n, 0}$ satisfies (3.7) with $n=n+1$ for $n \in \mathbb{Z}_{m-1}^{+}$from (3.12).

We now prove the second result. For $j \in \mathbb{Z}_{2 m}, i \in \mathbb{Z}_{j}, t \in I$ and $z \in \Omega$, we have that

$$
\begin{aligned}
& R_{n, 0}^{(i, j-i)}(z, t) \\
& =\int_{0}^{\infty} \mathcal{D}_{t}^{j-i}\left(L_{0,0}^{(i, 0)}(z, h(t, \tau)) L_{n-1,1}(h(t, \tau), t) h^{(0,1)}(t, \tau)\right) \mathrm{e}^{-\kappa \tau} \mathrm{d} \tau .
\end{aligned}
$$


It follows from the assumptions on $L_{0,0}, L_{n-1,1}$ and $g$ that $R_{n, 0}$ satisfies Assumption 3.13. This finishes the proof.

We are now ready to prove the properties of $L_{n, 0}$ and $L_{n, 1}$ for $n \in \mathbb{N}_{0}$.

Lemma 3.16. If $g \in C^{2 m+1}(D)$ satisfies Assumption 3.5 for some $m \in \mathbb{N}$ and $K_{\kappa} \in \widetilde{C}_{\kappa, 0}^{2 m}(D)$ satisfies Assumption 3.6, then $L_{n-1,0}$, $L_{n-1,1} \in C_{\kappa, 0}^{2 m}(D)$ are less oscillatory kernels of order $n$ for $n \in \mathbb{Z}_{m}^{+}$.

Proof. According to the assumption on $K_{\kappa}$, we have that $L_{0,0}$ and $L_{0,1} \in C_{\kappa, 0}^{2 m}(D)$ are less oscillatory kernels of order 1 . The results of this lemma may be proved by induction $n$ with the help of the definitions of (3.10), (3.11), Lemma 3.14 and Lemma 3.15.

We next present a property of the iterated integral operators $\mathcal{V}_{\kappa}^{n}$ for $n \in \mathbb{Z}_{m}^{+}$in the following theorem.

Theorem 3.17. If $g \in C^{2 m+1}(D)$ satisfies Assumption 3.5 for some $m \in \mathbb{N}$ and $K_{\kappa} \in \widetilde{C}_{\kappa, 0}^{2 m}(D)$ satisfies Assumption 3.6 , then the operator $\mathcal{V}_{\kappa}^{n}$ maps $C_{\kappa}^{m}(I)$ into $\widetilde{C}_{\kappa, m-n}^{m}(I)$ for $n \in \mathbb{Z}_{m}^{+}$.

Proof. For $n \in \mathbb{Z}_{m}^{+}$, we prove this result by using the decomposition for $\mathcal{V}_{\kappa}^{n}$, which is $\mathcal{V}_{\kappa}^{n}=\mathcal{W}_{\kappa}\left[L_{n-1,0}\right]+\mathcal{W}_{0}\left[L_{n-1,1}\right]$. This, together with Lemma 3.10 and Lemma 3.16 yields the conclusion.

We now state the main result of this section: it describes the structure of the solution of the VIEs.

Theorem 3.18. If $g \in C^{2 m+1}(D)$ satisfies Assumption 3.5 for some $m \in \mathbb{N}, K_{\kappa} \in \widetilde{C}_{\kappa, 0}^{2 m}(D)$ satisfies Assumption 3.6, and $f \in \widetilde{C}_{\kappa, 0}^{m}(I)$ satisfies Assumption 3.7, then the solution $u$ of the VIE (2.1) belongs to the space $\widetilde{C}_{\kappa, 0}^{m}(I)$.

Proof. We obtain the desired result by applying equation (3.1) for $n=m$ with the assumptions on $K_{\kappa}, f$ and $g$.

To close this section, we observe that a nonlinear oscillator satisfying the assumptions introduced in this section is $g_{0}(x):=(x+1)^{2}(x \in I)$; here we could choose $\Omega:=\mathbb{C} \backslash[-1,-\infty)$. We shall analyze the 
oscillation structure of solutions to VIEs with such nonlinear oscillators in a future paper.

\section{The oscillation structure of the solution to the oscillatory} VIDEs. In this section, we study the oscillatory structure of Volterra integro-differential equations with the form

$$
u^{\prime}(t)=a(t) u(t)+f(t)+\int_{0}^{t} K(t, s) \mathrm{e}^{\mathrm{i} \kappa g(t, s)} u(s) \mathrm{d} s, \quad \text { for } t \in I,
$$

and $u(0)=u_{0}$, where $g$ and $K \in C(D)$ are assumed to be independent of the wavenumber $\kappa>1$. We assume that $g(t, s):=g_{0}(t)-g_{0}(s)$ for $(t, s) \in D$, where $g_{0} \in C^{1}(I)$ satisfying $g_{0}^{\prime}(t) \neq 0$ does not depend on $\kappa$ for $t \in I$. We first derive the VIE. We now prove that the representation of the solution to (4.1) by the differential resolvent kernel contains the oscillatory component of the given kernel. The oscillatory structure of the solution to (4.1) is then analyzed by rewriting the VIDE as a second-kind VIE.

We first show that the differential resolvent kernel of (4.1) inherits the highly oscillatory term $\mathrm{e}^{\mathrm{i} \kappa g}$.

Theorem 4.1. If $a, f \in C(I)$ and $K \in C(D)$, then, for any initial value $u_{0} \in \mathbb{R}$, the $\operatorname{VIDE~(4.1)~has~a~unique~solution~} u \in C^{1}(I)$. Moreover, there exists a unique function $r \in C^{1}(D)$ such that $u$ can be written in the form

$$
u(t)=r_{\kappa}(t, 0) u_{0}+\int_{0}^{t} r_{\kappa}(t, s) f(s) \mathrm{d} s, \quad \text { for } t \in I,
$$

where $r_{\kappa}(t, s):=r(t, s) \mathrm{e}^{\mathrm{i} \kappa g(t, s)}$ for $(t, s) \in D$.

Proof. The proof is carried out along the lines of the method used in [3]. We first multiply both sides of (4.1) by $\mathrm{e}^{-\mathrm{i} \kappa g_{0}(t)}$ for $t \in I$; this yields

$$
\begin{aligned}
& u_{\kappa}^{\prime}(t)=\left(a(t)-\mathrm{i} \kappa g_{0}^{\prime}(t)\right) u_{\kappa}(t)+f_{\kappa}(t)+\int_{0}^{t} K(t, s) u_{\kappa}(s) \mathrm{d} s(t \in I), \\
& u_{\kappa}(0)=u_{0} \mathrm{e}^{-\mathrm{i} \kappa g_{0}(0)}
\end{aligned}
$$


where, for $t \in I, u_{\kappa}(t)=u(t) \mathrm{e}^{-\mathrm{i} \kappa g_{0}(t)}$ and $f_{\kappa}(t)=f(t) \mathrm{e}^{-\mathrm{i} \kappa g_{0}(t)}$. This is a linear first-order VIDE for $u_{\kappa}$. According to classical Volterra theory [2], we obtain that the unique solution of (4.3) $u_{\kappa} \in C^{1}(I)$ has the form

$$
u_{\kappa}(t)=r(t, 0) u_{\kappa}(0)+\int_{0}^{t} r(t, s) f_{\kappa}(s) \mathrm{d} s, \quad \text { for } t \in I,
$$

where $r \in C^{1}(D)$ denotes the differential resolvent kernel of the VIDE (4.3). Multiplying both sides of (4.4) by $\mathrm{e}^{\mathrm{i} \kappa g_{0}(t)}$ for $t \in I$ yields the conclusion.

Note that Theorem 4.1 does not tell us whether or not $r$ is highly oscillatory. However, for the following special case, the differential resolvent kernel $r$ of the VIDE (4.3) is independent of $\kappa$.

Corollary 4.2. If $a, f \in C(I), K \in C(D)$ and $a=\mathrm{i} \kappa g_{0}^{\prime}$, then the differential resolvent kernel $r_{\kappa}$ of (4.1) has the form $r_{\kappa}=r \mathrm{e}^{\mathrm{i} \kappa g}$, where $r \in C^{1}(D)$ is independent of $\kappa$.

Proof. We show that the differential resolvent kernel of the VIDE (4.3) for $u_{\kappa}$ is independent of $\kappa$. It follows from [2] that the differential resolvent kernel of (4.3) is defined by

$$
r(t, s):=1+\int_{s}^{t} Q(t, v) \mathrm{d} v, \quad(t, s) \in D,
$$

where $Q$ satisfies the resolvent equation

$$
Q(t, s)=H(t, s)+\int_{s}^{t} Q(t, v) H(v, s) \mathrm{d} v, \quad(t, s) \in D,
$$

with

$$
H(t, s)=a(s)-\mathrm{i} \kappa g_{0}^{\prime}(s)+\int_{s}^{t} K(v, s) \mathrm{d} v, \quad(t, s) \in D .
$$

By the assumption on the function $a$, we see that

$$
H(t, s)=\int_{s}^{t} K(v, s) \mathrm{d} v \quad \text { for }(t, s) \in D
$$

is independent on $\kappa$. This ensures that $Q$ is independent of $\kappa$. The assertion that $r$ is independent of $\kappa$ from (4.5) then follows. 
We now study the oscillatory structure of the solution to the VIDE (4.1) by rewriting the initial-value problem (4.1) as a second-kind VIE, namely

$$
u(t)=\tilde{f}(t)+\int_{0}^{t} K_{\kappa}(t, s) u(s) \mathrm{d} s, \quad \text { for } t \in I,
$$

where, for $(t, s) \in D$,

$$
\begin{aligned}
\tilde{f}(t) & =u_{0}+\int_{0}^{t} f(s) \mathrm{d} s, \\
K_{\kappa}(t, s) & =a(s)+\int_{s}^{t} K(v, s) \mathrm{e}^{\mathrm{i} \kappa g(v, s)} \mathrm{d} v,
\end{aligned}
$$

and then using the analogous results for VIEs presented in the previous section. Let $R_{\kappa}$ denote the resolvent kernel of the VIE (4.6). The differential resolvent kernel for (4.1) is then given by

$$
r_{\kappa}(t, s):=1+\int_{s}^{t} R_{\kappa}(t, v) \mathrm{d} v, \quad(t, s) \in D .
$$

We shall derive the oscillatory structures of the resolvent kernel and the solution to the highly oscillatory VIEs (4.6) under appropriate assumptions on $g, a, f$ and $K$. We then obtain the oscillatory structure of the solution to the VIDEs (4.1).

Corollary 4.3. If $K$ and $g \in C^{m}(D)$ for a fixed integer $m \in \mathbb{N}$ are independent of $\kappa$ and $a \in C_{\kappa}^{m}(I)$, then $r_{\kappa} \in C_{\kappa}^{m}(D)$, where $r_{\kappa}$ is the differential resolvent kernel of (4.1).

Proof. We prove this result by first verifying that the kernel $K_{\kappa}$ defined by (4.7) belongs to the space $C_{\kappa}^{m}(D)$ and by then applying Theorem 2.8 with the definition (4.8) of the differential resolvent kernel of (4.1) to obtain the conclusion of this corollary. We only estimate the bound of the derivatives of $K_{\kappa}$. It follows from (4.7) that $K_{\kappa}^{(1,0)}=K \mathrm{e}^{\mathrm{i} \kappa g}$,

$$
K_{\kappa}^{(1+j, 0)}=\left(K \mathrm{e}^{\mathrm{i} \kappa g}\right)^{(j, 0)}\left(j \in \mathbb{Z}_{m-1}\right),
$$


while, for $j \in \mathbb{Z}_{m},(t, s) \in D$,

$$
\begin{aligned}
K_{\kappa}^{(0, j)}(t, s)= & a^{(j)}(s)+\int_{s}^{t}\left(K(v, s) \mathrm{e}^{\mathrm{i} \kappa g(v, s)}\right)^{(0, j)} \mathrm{d} v \\
& -\sum_{i \in \mathbb{Z}_{j-1}}\left[\left.\left(K(v, s) \mathrm{e}^{\mathrm{i} \kappa g(v, s)}\right)^{(0, i)}\right|_{v=s}\right]^{(j-i-1)} .
\end{aligned}
$$

Then, for $j \in \mathbb{Z}_{m}^{+}$and $i \in \mathbb{Z}_{j}^{+}$, we have that $K_{\kappa}^{(i, j-i)}=\left(K \mathrm{e}^{\mathrm{i} \kappa g}\right)^{(i-1, j-i)}$. These equations, together with the assumptions stated in this corollary, yield that $K_{\kappa} \in C_{\kappa}^{m}(D)$. This completes the proof.

Note that the conclusion of Corollary 4.3 is valid for any nonlinear oscillator $g$.

We next state the main result of this section.

Theorem 4.4. If $g \in C^{2 m+1}(D)$ satisfies Assumption 3.5 for some $m \in \mathbb{N}, a \in C_{\kappa, 0}^{2 m}(I)$ satisfies Assumption 3.3 , and $f \in \widetilde{C}_{\kappa, 0}^{m}(I)$ satisfies Assumption 3.7, then the solution $u$ of the VIDEs (4.1) belongs to the space $\widetilde{C}_{\kappa, 0}^{m}(I)$.

Proof. We prove this result by using the results for the VIE (4.6). According to Theorem 3.18, it suffices to verify that $\widetilde{f} \in \widetilde{C}_{\kappa, 0}^{m}(I)$ satisfies Assumption 3.7, and $K_{\kappa} \in \widetilde{C}_{\kappa, 0}^{2 m}(D)$ satisfies Assumption 3.6, where $\tilde{f}$ and $K_{\kappa}$ are defined in (4.7). The assertion for $\tilde{f}$ can be proved as that in Theorem 3.9, and we omit the proof. According to the decomposition of the oscillatory functions of the form (3.3), we have that, for $(t, s) \in D$,

$$
\begin{aligned}
K_{\kappa}(t, s)= & -\mathrm{e}^{\mathrm{i} \kappa g(t, s)} \int_{0}^{\infty} K(h(t, \tau), s) \mathrm{e}^{-\kappa \tau} \mathrm{d} \tau \\
& +\int_{0}^{\infty} K(h(s, \tau), s) \mathrm{e}^{-\kappa \tau} \mathrm{d} \tau+a(s),
\end{aligned}
$$

where $h(t, \tau):=g_{0}^{-1}\left(g_{0}(t)+\mathrm{i} \tau\right)$ for $t \in I$ and $\tau \in[0, \infty)$. This, together with the assumption of the theorem, yields the desired result for $K_{\kappa}$. 
To close this section, we use three examples to illustrate the form of the differential resolvent kernel of the VIDEs (4.1) for some special cases.

Example 4.5. We choose $a(t)=\mathrm{i} \kappa, g_{0}(t)=t$ and $K(t, s)=\lambda$ for $(t, s) \in D$. Equation (4.3) reduces to

$$
u_{\kappa}^{\prime}(t)=f_{\kappa}(t)+\lambda \int_{0}^{t} u_{\kappa}(s) \mathrm{d} s, \quad \text { for } t \in I, \text { and } u_{\kappa}(0)=u_{0} .
$$

$u_{\kappa}$ satisfies the second-kind Volterra integral equation

$$
u_{\kappa}(t)=\widehat{f}(t)+\int_{0}^{t} K_{\kappa}(t, s) u_{\kappa}(s) \mathrm{d} s, \quad t \in I,
$$

where

$$
\widehat{f}(t)=u_{0}+\int_{0}^{t} f_{\kappa}(s) \mathrm{d} s
$$

and $K_{\kappa}(t, s)=\lambda(t-s)$ is independent of $\kappa$. According to Corollary 4.2, we obtain that the resolvent kernel has the form $r_{\kappa}=r \mathrm{e}^{\mathrm{i} \kappa g}$ with $r$ independent of $\kappa$.

Example 4.6. We choose $a(t)=\lambda /(\mathrm{i} \kappa), g_{0}(t)=t$ and $K(t, s)=\lambda$ for $(t, s) \in D$. We have that $K_{\kappa}(t, s)=\lambda /(\mathrm{i} \kappa) \mathrm{e}^{\mathrm{i} \kappa(t-s)}$. The solution of (4.6) has the form

$$
u(t)=\tilde{f}(t)+\frac{\lambda}{\mathrm{i} \kappa} \int_{0}^{t} \mathrm{e}^{\mathrm{i}\left(\lambda \kappa^{-1}+\kappa\right)(t-s)} \tilde{f}(s) \mathrm{d} s, \quad \text { for } t \in I .
$$

The differential resolvent kernel is, for $(t, s) \in D$,

$$
r_{\kappa}(t, s)=1+\lambda\left(1-\mathrm{e}^{\mathrm{i}\left(\lambda \kappa^{-1}+\kappa\right)(t-s)}\right) /\left(\lambda+\kappa^{2}\right) .
$$

Example 4.7. We consider the kernel $K_{\kappa}$ of (4.6) satisfying $K_{\kappa}=$ $W \mathrm{e}^{\mathrm{i} \kappa g}$, with $W \in C(D)$ independent of $\kappa$. The resolvent kernel associated with $K_{\kappa}$ then has the form

$$
R_{\kappa}(t, s)=R(t, s) \mathrm{e}^{\mathrm{i} \kappa g(t, s)} \quad \text { for }(t, s) \in D,
$$

where $R$ is the resolvent kernel associated with $W$. We obtain that the differential resolvent kernel of (4.1) has the form (4.5). 
5. Concluding remarks. We analyze in this paper the oscillatory properties of solutions of VIEs and VIDEs with highly oscillatory kernels, where the oscillatory component of the given kernel is a product of a non-oscillatory smooth function and a typical known oscillatory function with a nonlinear separable oscillator. The oscillatory components of the solution are derived by using the iterated integral operators to represent the solution. We observe that the oscillatory structure of the solutions is similar to that of the given kernel of the VIEs and VIDEs. In future work, we will study the oscillatory properties of solution of VIEs and VIDEs with highly oscillatory kernels for the case where the given kernel has a weak singularity and a more general (nonlinear) oscillator. Our results will also form the basis for designing efficient numerical schemes (e.g., collocation and Galerkin methods) for approximating the solutions of VIEs and VIDEs with highly oscillatory kernels (in analogy to $[8,9,13,14,29])$.

\section{REFERENCES}

1. H. Brunner, A survey of recent advances in the numerical treatment of Volterra integral and integro-differential equations, J. Comp. Appl. Math. 8 (1982), 213-229.

2. Collocation methods for Volterra integral and related functional equations, Cambridge University Press, Cambridge, 2004.

3. _ On Volterra integral operators with highly oscillatory kernels, Discr. Cont. Dynam. Syst. 34 (2014), 915-929.

4. H. Brunner, A. Iserles and S.P. Nørsett, The computation of the spectra of highly oscillatory Fredholm integral operators, J. Int. Equat. Appl. 23 (2011), 467519 .

5. _ The spectral problem for a class of highly oscillatory Fredholm integral operators, IMA J. Numer. Anal. 30 (2010), 108-130.

6. H. Brunner, A. Pedas and G. Vainikko, The piecewise polynomial collocation method for nonlinear weakly singular Volterra equations, Math. Comp. 68 (1999), 1079-1095.

7. P.J. Davis and P. Rabinowitz, Methods of numerical integration, Academic Press, New York, 1984.

8. V. Domínguez, I.G. Graham and T. Kim, Filon-Clenshaw-Curtis rules for highly oscillatory integrals with algebraic singularities and stationary points, SIAM J. Numer. Anal. 51 (2013), 1542-1566.

9. V. Domínguez, I.G. Graham and V.P. Smyshlyaev, Stability and error estimates for Filon-Clenshaw-Curtis rules for highly oscillatory integrals, IMA J. Numer. Anal. 31 (2011), 1253-1280. 
10. G. Gripenberg, S.-O. Londen and O. Staffans, Volterra integral and functional equations, Cambridge University Press, Cambridge, 1990.

11. D. Huybrechs and S. Vandewalle, On the evaluation of highly oscillatory integrals by analytic continuation, SIAM J. Numer. Anal. 44 (2006), 1026-1048.

12. The construction of cubature rules for multivariate highly oscillatory integrals, Math. Comp. 76 (2007), 1955-1980.

13. A. Iserles and S.P. Nørsett, Efficient quadrature of highly oscillatory integrals using derivatives, Proc. Amer. Math. Soc. 461 (2005), 1383-1399.

14. Quadrature methods for multivariate highly oscillatory integrals using derivatives, Math. Comp. 75 (2006), 1233-1258.

15. W.P. Johnson, The curious history of Faà di Bruno's formula, Amer. Math. Month. 109 (2002), 217-234.

16. S.I. Kabanikhin and A. Lorenzi, Identification problems of wave phenomena: Theory and numerics, VSP, Utrecht, 1999.

17. D. Levin, Analysis of a collocation method for integrating rapidly oscillatory functions, J. Comp. Appl. Math. 78 (1997), 131-138.

18. , Procedures for computing one- and two-dimensional integrals of functions with rapid irregular oscillations, Math. Comp. 38 (1982), 531-538.

19. Y. Ma and Y. Xu, Computing highly oscillatory integrals, in revision (2015).

20. R.K. Miller, Nonlinear Volterra integral equations, Benjamin, Menlo Park, CA, 1971.

21. S. Olver, Fast, numerically stable computation of oscillatory integrals with stationary points, BIT Numer. Math. 50 (2010), 149-171.

22. Moment-free numerical approximation of highly oscillatory functions, IMA J. Numer. Anal. 26 (2006), 213-227.

23. J. Riordan, An introduction to combinatorial analysis, Wiley, New York, 1958.

24. S. Roman, The formula of Faà di Bruno, Amer. Math. Month. 87 (1980), 805-809.

25. E. Stein, Harmonic analysis: Real-variable method, orthogonality, and oscillatory integral, Princeton, New Jersey, 1993.

26. F. Ursell, Integral equations with a rapidly oscillating kernel, J. Lond. Math. Soc. 44 (1969), 449-459.

27. H. Wang and S. Xiang, Asymptotic expansion and Filon-type methods for Volterra integral equation with a highly oscillatory kernel, IMA J. Numer. Anal. 31 (2011), 469-490.

28. Y. Wang and Y. Xu, Oscillation preserving Galerkin Methods for Fredholm integral equations of the second kind with oscillatory kernels, in revision (2015).

29. S. Xiang, Efficient Filon-type methods for $\int_{a}^{b} f(x) \exp \{\mathrm{i} \omega g(x)\} \mathrm{d} x$, Numer. Math. 105 (2007), 633-658.

30. S. Xiang and H. Brunner, Efficient methods for Volterra integral equations with highly oscillatory Bessel kernels, BIT Numer. Math. 53 (2013), 241-263. 
31. S. Xiang, Y.J. Cho, H. Wang and H. Brunner, Clenshaw-Curtis-Filon-type methods for highly oscillatory Bessel transforms and applications, IMA J. Numer. Anal. 31 (2011), 1281-1314.

Department of Mathematics, Hong Kong Baptist University, Hong Kong, P.R. China and Department of Mathematics \& Statistics, Memorial University of Newfoundland, St. John's, NL A1C 5S7, Canada

Email address: hbrunner@math.hkbu.edu.hk

Guangdong Provincial Key lab of Computational Science, School of Applied Computational Science and School of Data and Computer Science, Sun Yat-Sen University, Guangzhou 510275, P.R. China

\section{Email address: mayy007@foxmail.com}

Guangdong Provincial Key lab of Computational Science, School of Applied Computational Science and School of Data and Computer Science, Sun Yat-sen University, Guangzhou 510275, P.R. China and Professor Emeritus of Mathematics, Syracuse University, Syracuse, NY 13244

Email address: yxu06@syr.edu, xuyuesh@mail.sysu.edu.cn 